\title{
JOINTS ORIENTATION DISTRIBUTIONS IN SEDIMENTARY ROCKS
}

\author{
T. P. BELOUSOV, SH. A. MUKHAMEDIEV, S. F. KURTASOV \\ Institute of Physics of the Earth, B. Gruzinskaya str., 10, Moscow, \\ 123810, Russia, Institute of Geosystems, Varshavskoye h., \\ 8, Moscow, 113105, Russia
}

(Received 20 June 1995)

\begin{abstract}
The results of several years (1980-1994) field observations of rock joints at the former USSR territory are integrated. New methods of data processing and interpretation are proposed and developed. With the local data set for an exposure we connect the symmetric positively defined second order tensor $J$ characterizing the density and relative position of joint systems as well as their orientation in space. The complete set of tensors $J$ for any region depicted in appropriate manner gives the integral "portrait" of tectonic fracturing of the region. The regular changes of such integral characteristics for Great Caucasus as an example are determined.
\end{abstract}

KEY WORDS: Rock fracturing, joint orientation distribution, tensor characteristic.

Fracturing, one of the most significant characteristics of the upper crust is developed everywhere in rocks of various genesis and age. It is observed both at natural outcrops and walls of various minings. Cracks are not chaotic and ever exhibit regularity revealed as quasiperiodic and quasiparallel systems. The systems can often be observed visually but occasionally they can be found out only as a result of measured data processing. We examined fracturing of rocks with the view of fundamental study in meso-scale divisibility of sedimentary rocks as well as solution of two specific problems, namely reconstruction of crustal paleostress axes in seismically active regions and investigation of reservoir properties of rocks in oil-production regions.

\section{LOCAL DISTRIBUTIONS OF JOINT ORIENTATIONS}

Orientation of an individual tectonic crack (joint) is defined by any unit vector $\mathbf{n}$ of a normal directed toward the upper hemisphere. Whenever a quite representative set of orientations of $N$ joints is determined, it is described by a "cloud" (cluster) of points on a stereographic projection. Figure 1a shows relevant example of 100 pole orientations of normals $\mathbf{n}_{\mathbf{i}}$ to joint planes measured in a limestone bed in the Beshtau Mountain, Northern Pre-Caucasus area. This cloud is a random sample of a continuous orientation function. The latter is to be reconstructed from experimental data. The distribution 


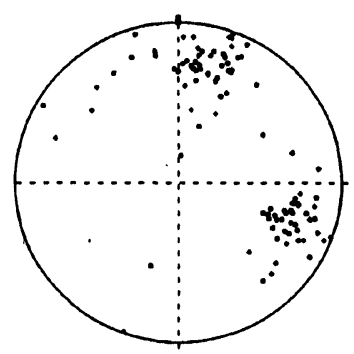

a

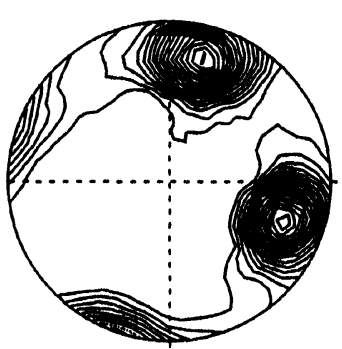

d

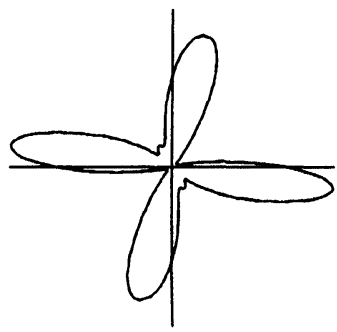

b

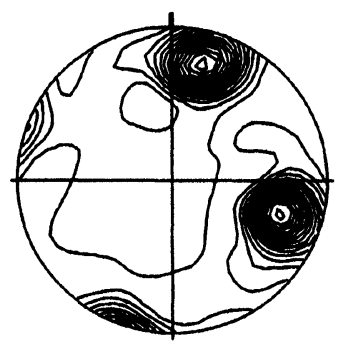

e

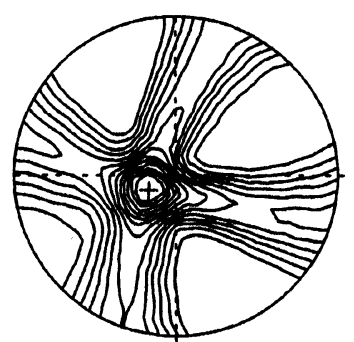

Figure 1 Joint orientation distribution in the Late Cretaceous limestone bed (Beshtau Mountain, Northern Pre-Caucasus). a - joint poles; $\mathrm{b}$ - continuous pole distribution, cell method; $\mathrm{c}$ - continuous pole distribution, Gaussian smoothing; $\mathrm{d}$ - one-dimension azimuth representation; $\mathrm{c}$ - correction of continuous distribution for bed rotation; $f$ - continuous distribution of joint planes.

function of joint orientations $\rho(\mathbf{n})$ represents the density of probability that a normal to a joint plane would coincide with various orientations of $\mathbf{n}$ in space.

A continuous distribution of $N$ orientations determined from experimental data can be reconstructed, for instance, by dividing a unit sphere into elementary cells and counting up the number of points within each cell. After the values of $\rho(\mathbf{n})$ are found throughout the stereogram area, contour isolines of pole density are drawn with the purpose of quantitative description of a joint orientation distribution and its graphic visualization. A theoretical pole diagram corresponding to the pole "cloud" of Figure $1 \mathrm{a}$ is shown in Figure 1b.

However we would improve results if, instead of simple counting-up of points within a cell, each point is represented by a continuous bell-shaped (Gaussian) function $\rho^{G}(\mathbf{n}$, $\left.\mathbf{n}_{\mathbf{i}}, \omega\right)$ with dispersion $\omega$ centered at $\mathbf{n}_{i}$ (Bunge, 1969). The dispersion parameter $\omega$ is chosen from the condition that individual peaks should overlap but, on the other hand, essential features of the distribution should not be smoothed more than is necessary (Figure 1c).

A one-dimensional azimuth representation of the orientation distribution (rose diagram) can be obtained by projecting joint poles $\mathbf{n}_{\mathbf{i}}$ onto a bed plane. We used only one of the available methods, namely Gaussian smoothing in order to calculate rose diagrams. An example of a relevant distribution is illustrated in Figure 1d.

Orientation distributions could undergo multiple rotations during the geologic history. Their original position is of interest for some applications, e.g. for reconstruction of principal axes of paleostresses. Therefore it is important that the distribution would be corrected for rotation of bedding layers arisen due to folding processes. An example 
of a corrected distribution is shown in Figure 1e where the normal to a bed plane was placed at the center of the projection.

Beside the description of the joint pole distribution $\rho(\mathbf{n})$, of interest are probability distributions of joint plane orientations $\gamma(\mathbf{n})$. This alternative approach to description of fracturing can be advantageous in studying reservoir properties of rocks. In order to estimate the distribution $\gamma(\mathbf{n})$, the number of joint plane intersections is counted up for measured joints having a conical solid angle $\Omega$. A joints plane distribution thus obtained is illustrated in Figure $1 \mathrm{f}$.

\section{INTEGRAL DISTRIBUTIONS OF JOINT ORIENTATIONS}

If general features of fracturing are studied which characterize a concrete region or rocks of specific age and composition in this region, then data measured at several observational points can be efficiently represented as a general distribution of orientations that will be referred here to as a summary one. Such distributions allow one to reveal both general strikes of joint systems that are characteristic of a region under consideration and relative density of joints in the systems. There are two means by which original local distributions of orientations can be summarized:

1. Bedding elements of joint planes in a general data set are represented by values measures in situ.

2. Data measured at any observational point are first corrected for rotation of beds.

Analysis of summary distributions in active regions shows that the fracturing pattern becomes essentially more ordered if a distribution is obtained for layers rotated to their original horizontal position. In this way a stereogram always exhibits distinct maxima of joint pole density even if they are not recognizable for summary distributions calculated without correction for rotation of beds. The maxima are located at margins of stereograms, which corresponds to the subvertical orientation of generalized joint systems after rotation of beds. Two maxima with the angular distance $90^{\circ} \mathrm{C}$ between them are identified most often. This indicates the presence of two orthogonal generalized systems of joints (Figure 2). Ordering resulting from the correction of local distributions for rotation of beds is suggestive of the fact that major fracture systems in folded regions arise in originally horizontal beds.
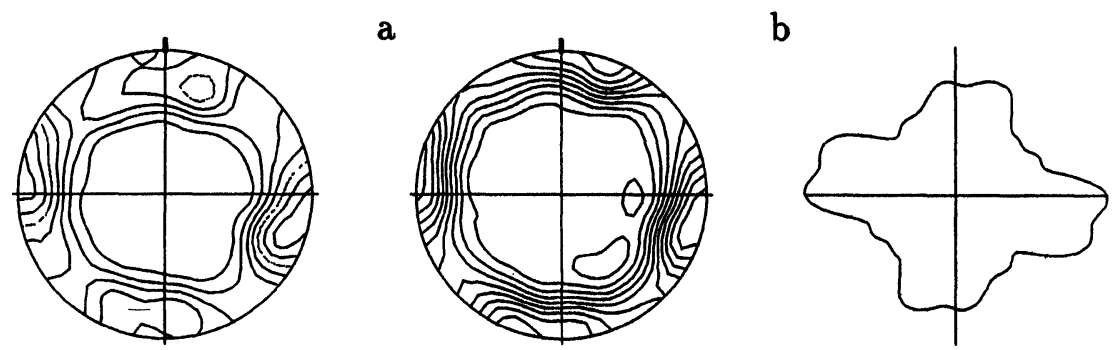

C

Figure 2 Summary distributions for 3860 joints in Cretaceous rocks of Great Caucasus. a - in situ measurements; $b$ - correction of local distributions for bed rotation; c - azimuth representation of joint poles after correction. 
Although various stereographic projections of joint distributions admit the visual representation, possibilities of their analysis are quite limited. It is desirable to describe a joint system with some quantitative characteristic but a scalar or vector quantity is clearly inadequate for this purpose. Thus volume joint density cannot give any information about angular distribution of joints, and if a direction only of one of joint systems is specified then any information on other systems is lost. A second-rank tensor is a simplest object that allows for mutual position and spatial orientations of joint systems as well as relative density of joints in a system.

We introduce a nonnegative symmetric tensor of second rank $\mathbf{J}$ normed to its first invariant:

$$
\mathbf{J}=a \sum_{i=1}^{N}\left(\mathbf{I}-\mathbf{n}_{i} \otimes \mathbf{n}_{i}\right), \quad a=1 / 2 N, \quad I_{1}(\mathbf{J})=1
$$

where $I$ is the unit tensor.

This is an inertia tensor of local orientation distribution with eigenvalues $J_{1}, J_{2}$ and $J_{3}$. The tensor $\mathbf{J}$ is characterized by five independent scalar quantities which can be taken as the invariants $S$ (intensity of the deviator of $\mathbf{J}$ ) and $\omega_{J}$ (an angle of eigenvalues ratio $0 \leq \omega_{J} \leq \pi / 3$ ) and three Euler angles that fix the tripod of principal axes of the tensor $\mathbf{J}$ in space.

Invariants of the tensor $\mathbf{J}$ are represented graphically in such a way that a point $\left(J_{1}\right.$, $J_{2}, J_{3}$ ) with similarly designated, mutually orthogonal axes in a three-dimensional space is projected orthogonally onto six points of the deviatoric plane $J_{1}+J_{2}+J_{3}=0$. The latter passes through the origin of coordinates and is inclined at equal angles to the axes $J_{1}, J_{2}$ and $J_{3}$. The invariants $\sqrt{2} S$ and $\omega_{J}$ constitute a natural system of polar coordinates in this plane: the intensity $S$ multiplied by $\sqrt{2}$ is a distance in the plane from a point projection to the origin of coordinates $J_{1}=J_{2}=J_{3}=0$, and the polar angle $\omega_{J}$ is measured from the negative continuation of the projection of the axes $J_{1}$, $J_{2}$ and $J_{3}$.

Points $\left(S, \omega_{J}\right)$ on the deviatoric plane correpond to tensors $\mathbf{J}$ at various points of observation in a region under consideration (or, alternatively, for rocks of a fixed age in the region) and their set gives a "portrait" of internal geometry of tectonic fracturing, typical of the region. We note that folding developed in the region does not affect this integral image since rotation of the tensor $\mathbf{J}$ does not affect the position of imaging points on the deviatoric plane.

We use two stereograms representing axis orientations of a set of tensors $\mathbf{J}$ for a given region: the first one (a) shows the cloud of points corresponding to emergence poles of the axis of a maximum eigenvalue $J_{1}$, and the second one (b) shows the rose diagram of the projection onto the horizontal plane of the axis $J_{2}$.

Figure 3 shows integral characteristics obtained by this method for Jurassic $(J)$, Cretaceous $(K)$, Paleogene $(P g)$ rocks in Great Caucasus as well as for Neogene $(N)$ rocks in North Pre-Caucasus. The following features are noteworthy.

- On the average, points characterizing older rocks on an individual "portrait" of a region (Figure 3c) are located closer to the origin of coordinates as compared with younger rocks. This indicates that older rocks contain additional joint systems due to which the tensor $\mathbf{J}$ of local distributions is closer to a spherical one. 


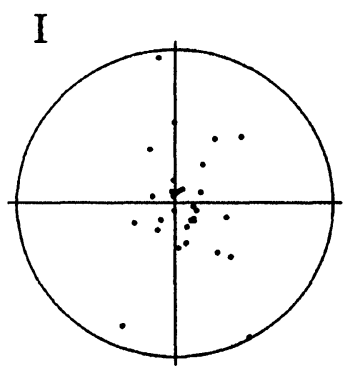

a

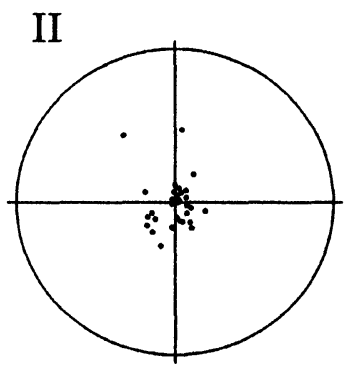

\section{III}
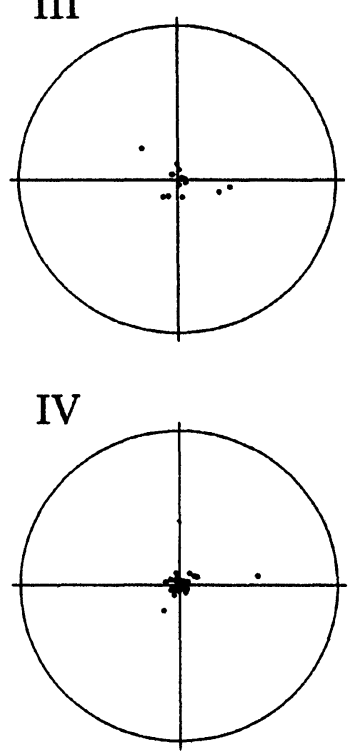

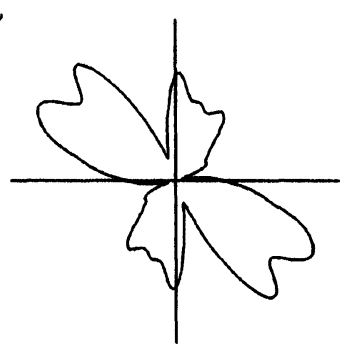

b
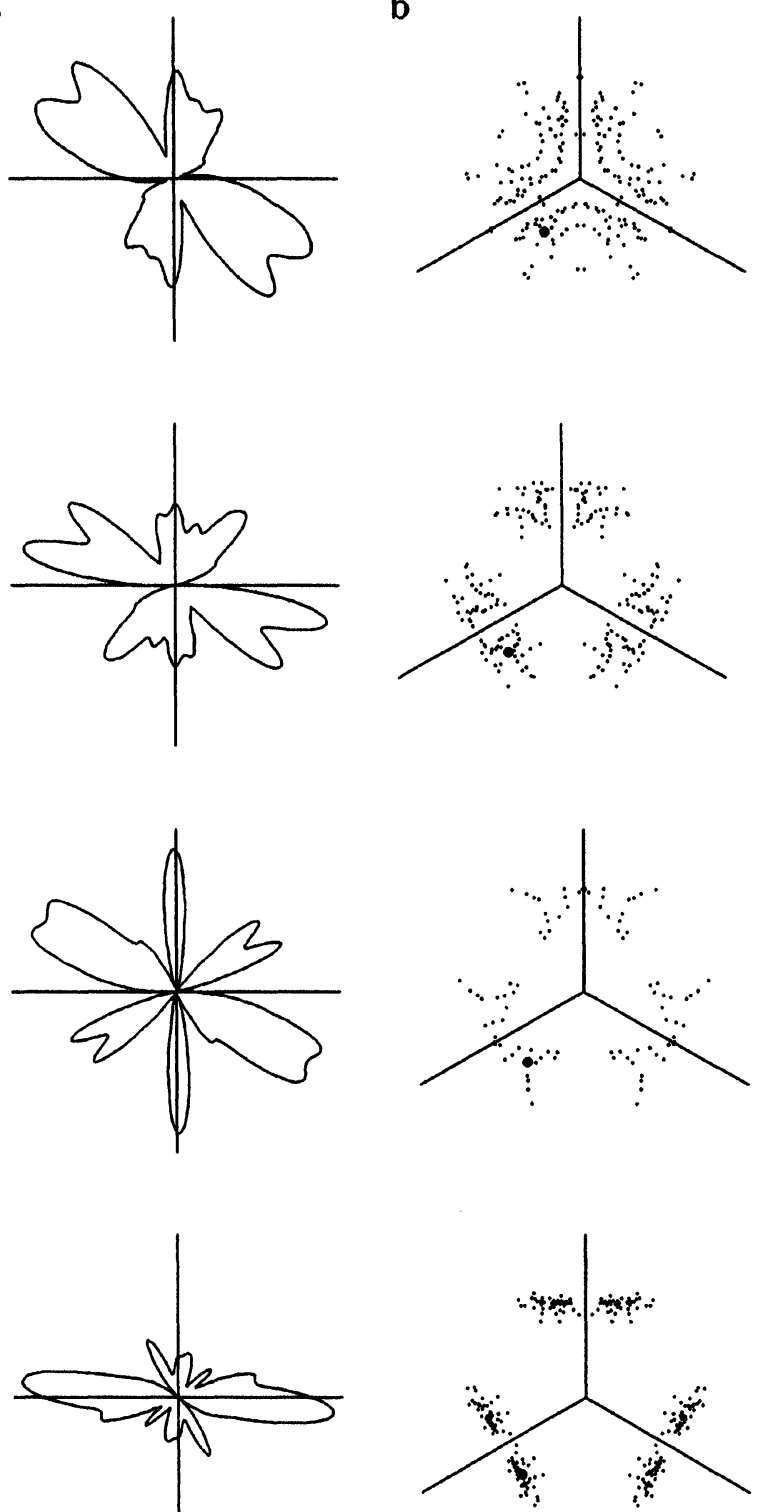

Figure 3 Integral characteristics of joint distributions for Great Caucasus and North Pre-Caucasus. a - stereogram of poles of $J_{1}$ axis; b - azimuth representation of $J_{2}$ axis; c - projections of eigenvalues $J_{1}, J_{2}, J_{3}$ onto deviatoric plane. I - Jurassic $(J)$ rocks, II - Cretaceous $(K)$ rocks, III - Paleogene $(P g)$ rocks, IV - Neogene $(N)$. 
- The younger the rocks are, the smaller the number of individual "portrait" points related to small angles of the eigenvalues ratio $\omega_{J}$ until states with $\omega_{J}<1$ vanish altogether. On the other hand, distributions of "disk" type become more pronounced for younger rocks.

- Pole "clouds" of the axis $J_{1}$ (Figure 3a) become less elongated with decreasing age. This indicates that amplitudes of bending deformations lower for younger rocks.

Rose diagrams of the axis $J_{2}$ are similar for the Jurassic and Early Cretaceous but their configuration changes drastically for Late Cretaceous and Paleogene rocks. Analysis of paleostresses also leads to the conclusion on a geodynamic rearrangement at the transition from the Early to Late Cretaceous (Belousov, Kurtasov, Mukhamediev, 1994).

Approaches suggested here were tested for rocks of various age and composition in a wide range of regions differed in their preneotectonic and neotectonic history. Results of measurements were incorporated to a computer data base. The base includes software for fracturing data processing, which enables calculation and analysis of joint orientation distributions as well as data interpretation on the basis of modern theoretical conceptions of processes in the Earth's crust. At the moment the data base contains information on more than 1000 points of observation in 14 regions of Eastern Europe and Central Asia. The data has been obtained by the authors in expeditions in 1980 1994. We note that even tentative application of the data base has shown its high efficiency in studying geological processes. The work was partly supported by the Russian Foundation of Fundamental Research under the grant N94-05-17371.

\section{References}

Belousov, T. P., Kurtasov S. F, and Mukhamediev, Sh. A. (1994). Methods of rock fracturing data processing and results of interpretation (in russian). Institute of Physics of the Earth, Moscow. Bunge, H. -J. (1969). Mathematische Methoden der Texturanalyse. Akademie-Verlag, Berlin. 